\title{
FLUORIDE RELEASE AND RERELEASE AFTER RECHARGING OF TWO HYBRID RESIN RESTORATIONS IN PRIMARY TEETH. “A COMPARATIVE IN VITRO STUDY”.
}

\author{
Rania A. Elshweekh ${ }^{1} B D S$, Niveen S. Bakry ${ }^{2} h D$, Dalia M. Talaat ${ }^{3} P h D$, \\ Dawlat M. Ahmed ${ }^{4} P h D$
}

\begin{abstract}
INTRODUCTION: Fluoride-releasing restorative materials can be used as a reservoir releasing small amounts of fluoride to the teeth over a long time.Giomer represents a new class of dental materials that uses the pre reacted glass technology (PRG) with excellent mechanical and esthetic properties.

OBJECTIVES: The aim of this study is to evaluate the fluoride release ability of Giomer (Beautifil II) compared to Compomer (Dyract XP) and their fluoride recharge ability after exposure to topical fluoride varnish.

MATERIALS AND METHODS: The sample consisted of 80 freshly extracted primary anterior teeth. Standardized buccal class V cavities were prepared. Sample was randomly divided into 2 groups: Group I: included 40 teeth restored with Beautiful II. Group II: included 40 teeth restored with Dyract XP following the manufacturer's guidelines. Fluoride release was evaluated in the artificial saliva on 1st, 7th, 14th and 21st days. After 21days of release, $5 \% \mathrm{NaF}$ varnish was applied on buccal surface of all specimens. The amount of fluoride release after recharge was measured in the artificial saliva after 1st, 7th, 14th and 21st days using fluorine ion- specific electrode. Data were analyzed using Friedman's two-way analysis of variance by ranks, Dunn-Sidak method, Mann-Whitney U test and Wilcoxon Signed Ranks to achieve the aim of the study.

RESULTS: Beautiful II released significantly higher amount of fluoride compared to Dyract XP in first 7 days at $\mathrm{p}<0.0125$. Dyract XP released significantly higher amount of fluoride after 14 and 21 days at $\mathrm{p}<0.0125$. After $5 \% \mathrm{NaF}$ varnish treatment there was no significant difference in fluoride re-release amount of both Beautiful II and Dyract XP at $\mathrm{p}>0.0125$.

CONCLUSIONS: Fluoride release (ppm) was found to be significantly higher in Beautiful II than Dyract XP in the first week. Giomer could be considered a suitable class $\mathrm{V}$ restoration of primary teeth in high caries risk children.
\end{abstract}

KEYWORDS: Giomer, Compomer, Class V, Fluoride release, Recharge, Primary teeth.

1. Bachelor of Dentistry, Faculty of Dentistry, Alexandria University, Alexandria, Egypt.

2. Professor of Pediatric Dentistry, Faculty of Dentistry, Alexandria University, Alexandria, Egypt.

3. Associate Professor of Pediatric Dentistry, Faculty of Dentistry, Alexandria University, Alexandria, Egypt.

4. Lecturer at Dental Biomaterials department, Faculty of Dentistry, Alexandria University,

Corresponding author:

E-mail: elshweekh86@gmail.com

\section{INTRODUCTION}

Over the last 30 years, dentistry has experienced a remarkable scientific advance regarding the improvement of restorative materials and techniques. Many different restorative materials were introduced to provide the best intraoral performance in terms of durability, aesthetics and symptom relief (1).

The addition of fluoride to restorative materials has attracted the attention of dental researchers and clinicians due to the possibility of their use as a reservoir releasing small amounts of fluoride, especially in patients with high caries risk (2). The story of the recognition and exploration of fluoride's ability to prevent caries occurrence or progression is long and interesting (3).

Fluoride has been known to have a role in the reduction of dental caries since early observations in the 1930s (4). It increases the tooth resistance to caries through different protective mechanisms such as biological and physicochemical.

Biologically, fluoride can interfere with pellicle and plaque formation. It also affects the metabolic activity of cariogenic bacteria and prevents it from secreting enzymes that ferment carbohydrates and subsequently decrease acid production. Moreover, fluoride plays a role in inhibition of microbial growth (5).

From the physicochemical point of view, fluoride reverse the demineralization process in the oral cavity and enhance the remineralization by replacing the hydroxyl groups in the upper layers of the hydroxypatite crystals to be fluoroappetite which results in a hard dental tissues with less solubility (6).

Glass ionomers were invented in the United Kingdom in 1969 and released commercially in the 1970s. This type of restoration has several advantages such as tooth color replication, biocompatibility ,fluoride ion release and uptake by enamel and dentin, coefficient of thermal expansion similar to that of tooth structure, and chemical bonding to both enamel and dentin (7). However, dentists did not widely accept these materials as a permanent restoration due to its susceptibility to dissolution during hardening, poor wear resistance and low fracture strengths, long setting times and unsatisfactory esthetics (8).

Hybrid materials combining the technologies of glassionomer and composites were developed to overcome the previous disadvantages of glass-ionomer cements. These hybrid materials mainly include Resin-modified Glass 
ionomer cements (RMGIC's), Compomers (Polyacidmodified composites) \& Giomers (9).

Compomers are esthetic materials that combine the benefits of traditional composite resins and those of glassionomer cements (10). Their popularity is due to their excellent handling with many advantages as; ease of placement with good aesthetics, not require mixing, easy to polish, less susceptible to dehydration with fluoride releasing ability and radiopacity (11). Unfortunately, when compared with composite, their physical properties are weaker (10), they show more marginal staining, matrix expansion due to water sorption, and in addition they require a bonding agent so they are technique sensitive (12).

A new approach in restorative dentistry has been the introduction of "Giomers" as hybrid aesthetic restorative materials. They contain both of the basic components of glass-ionomer cements and resins but they are not considered as compomers. They are placed in a separate category of composites known as pre-reacted glass-ionomer (PRG) composite (13). Beautifil II is a second generation giomer that uses modified surface pre-reacted glass ionomer (SPRG) filler technology, where only the surface of the fluoridated glass filler reacts with acid to form a thick siliceous hydrogel layer leaving inner unreacted glass core (14).

Giomers have excellent aesthetics, good color matching, smooth surface finish, decreased microleakage and clinical stability which introduce them as an acceptable choice for restoration of non-carious cervical lesions such as abrasion or erosion and class $\mathrm{V}$ cavities in primary or permanent teeth (15).

In 2011, Dhull et al (16), compared between Giomer and Compomer in fluoride releasing ability before and after daily topical fluoride application and concluded that, with or without topical application of the fluoridated dentifrice the fluoride release was significantly higher in Giomer than Compomer.

However, in 2015, Gui et al (17) evaluated the fluoride release and recharge of six restorative materials including Giomer and Compomer. They found that the two materials released comparable amount of fluoride.

Due to this contradiction between studies comparing Giomer (Beautifil II) and Compomer (Dyract XP) in fluoride release and recharge, the present study was conducted with the purpose of evaluating the fluoride release ability of Giomer compared to Compomer, and their fluoride recharge ability after exposure to topical fluoride varnish.

The first null hypothesis was that Giomer (Beautifil II) and Compomer (Dyract XP) would release same amount of fluoride ion. The second null hypothesis was that there would be no difference in the fluoride recharging ability of both restorative materials after exposure to topical fluoride varnish.

\section{MATERIALS AND METHODS}

The present in-vitro study was carried out in the Department of Pediatric and Preventive Dentistry, Alexandria University and the Institute of Graduate studies and Research, Environmental Studies Department, Alexandria University after the approval of the ethics committee.

The estimated sample size was 40 per group with $80 \%$ power $(\beta=20 \%)$ and at a significance $(\alpha=0.0125)(18)$. Total sample was 80 non carious anterior primary teeth extracted for orthodontic reasons or exfoliated, free from cracks or any developmental defects. Surfaces of all teeth included in the study were cleaned from debris and blood using fluoride free pumice and low speed handpiece. Teeth were stored in normal saline. Standard non-beveled buccal class V cavities were prepared following the cavity preparation guidelines for composite ( $3 \mathrm{~mm}$ wide, $2 \mathrm{~mm}$ long and $1 \mathrm{~mm}$ deep) in the cervical 1/3 of each tooth using a standard \# 330 diamond bur mounted at high speed with air/water cooled hand piece. For standardization purpose, the bur's length and a millimeter ruler were used to measure the dimensions of the cavity (19).

All prepared teeth were thoroughly cleaned with water and gently dried. Teeth were randomly divided into two groups according to restorative material used: Group I $(n=40)$ : was restored with Giomer (Beautifil II, Shofu, Kyoto, Japan). Group I I : was restored with Compomer (Dyract XP, Caulk/DENTSPLY). All steps were done following the manufacturer's instructions.

All restored teeth were stored in labeled plastic vials containing $1 \mathrm{ml}$ of artificial saliva of $\mathrm{pH}$ value was 7 , as the closest approximation to physiologic values for saliva in the oral cavity. Fluorine ion concentration in the artificial saliva was zero ppm. All the samples were kept in the incubator at $37^{\circ} \mathrm{C}$ for 24 hours.

\section{Fluoride release}

After 24 hours, the plastic vials were thoroughly shaken and then the teeth were removed, washed with $1 \mathrm{ml}$ of distilled water and returned into a new vial containing $1 \mathrm{ml}$ of fresh artificial saliva. Cumulative fluoride concentration in the artificial saliva was measured on $1^{\text {st }}, 7^{\text {th }}, 14^{\text {th }}$ and $21^{\text {st }}$ days.

\section{Fluoride re-release}

After 21 days of initial fluoride release, 5\% Sodium Fluoride varnish was applied on the buccal surface of all specimens in groups I and II using a disposable brush and allowed to dry for 5 minutes according to the manufacturer's instructions. Each tooth was stored in $1 \mathrm{ml}$ of fresh artificial saliva with zero $\mathrm{ppm}$ fluoride ion concentration and was incubated at $37^{\circ} \mathrm{C}$ for 24 hours. Cumulative fluoride re-release was measured on $1^{\text {st }}, 7^{\text {th }}$, $14^{\text {th }}$ and $21^{\text {st }}$ days. (Fig. 1)

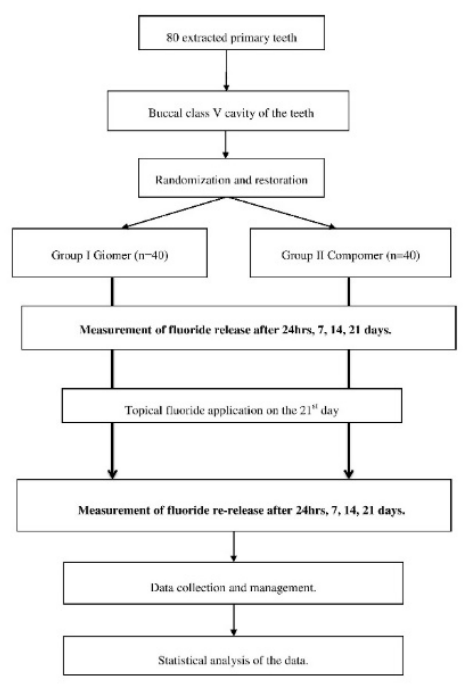

Figure 1: Flow Chart of the Study Design.

\section{Measurement of fluoride release and re-release}

Each collected artificial saliva sample was buffered with equal volume of Total Ionic Strength Adjustment Buffer (TISAB II) solution to control $\mathrm{pH}$ and prevent formation of fluoride complexes. 
Fluoride content was determined using fluorine ionspecific electrode (Fluoride electrode model 94-09BN, Orion Research Inc. products gp.529 Main st Boston MA 02129 USA).

The fluoride release concentrations were automatically displayed on the analyzer as millivoltage $(\mathrm{mV})$ readings which were entered into the computer using EXCEL software that mathematically established the part per million (ppm) values for each $\mathrm{mV}$ unit (20). The kappa statistic for intra-examiner reliability was 0.854 .

\section{Statistical analysis}

Data were collected and entered to the computer using SPSS (Statistical Package for Social Science) program for statistical analysis (ver 21). Comparisons of fluoride concentration at different time interval were evaluated using Friedman's twoway analysis of variance by ranks. Dunn-Sidak method was used for pair-wise comparison of each two time interval for each group. Mann-Whitney U test was used for comparison between two study groups at different intervals of time. Wilcoxon Signed Ranks test was used for comparing fluoride concentration before and after recharge in the same group. Correlation for $\mathrm{p}$ value was carried out using Bonferroni correction for multiple comparisons. Level of significance was set at $\mathrm{p}=0.0125$.

\section{RESULTS}

Pair wise comparison of fluoride concentrations in artificial saliva after teeth restoration with Giomer between different time intervals; 24hours, 7days, 14days, 21days indicated that there was a statistically significant difference when comparing every two intervals $(p=0.000)$ except between the fluoride release after 24 hours to 7 days and the release after 14 to 21 days ( $\mathrm{p}>0.0125)$. (Table I).

Table 1: Fluoride concentrations in artificial saliva after teeth restoration with Giomer in different time intervals.

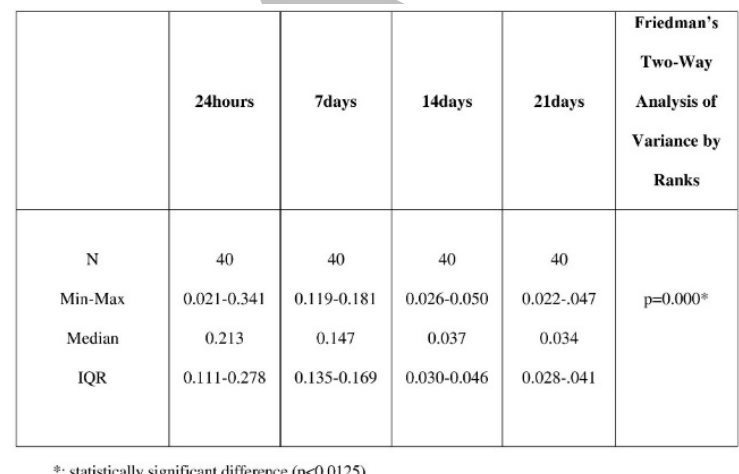

*: statistically significant difference $(p<0.0125)$

Pair wise comparison of fluoride concentrations in artificial saliva after teeth restoration with Compomer in different time intervals; 24hours, 7days, 14days, 21days indicated that there was statistically significant difference when comparing the fluoride release after 24 hrs to 7 days, 24 hrs to14 days and 24 hrs to 21 days $(\mathrm{p}<0.0125)$. However, comparisons between the release after 7 to 14 days, 7 to 21 days and 14 to 21days revealed that there was no statistically significant difference (p>0.0125). (Table II).
Table 2: Fluoride concentrations in artificial saliva after teeth restoration with Compomer in different time intervals.

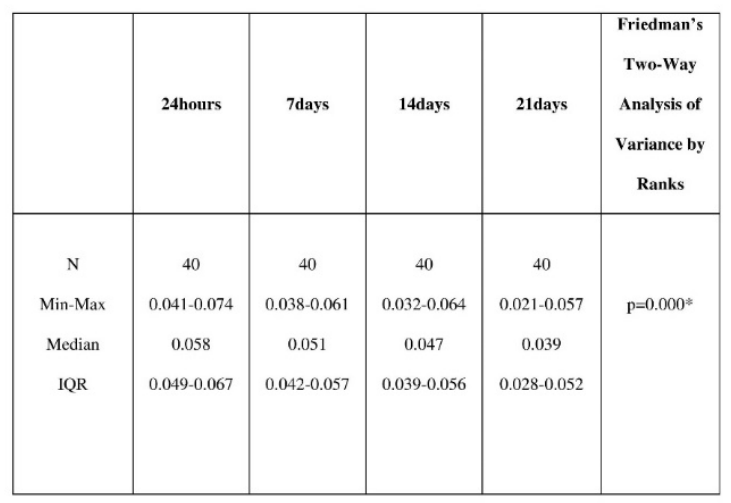

": statistically significant difference $(\mathrm{p}<0.0125)$

Comparison between groups I (Giomer) and II (Compomer) in each time interval separately revealed that Giomer was significantly higher than Compomer in fluoride release after 24hrs, 7days ( $p=0.000$ ). While Compomer was significantly higher than Giomer after 14days and 21 days of restoration ( $\mathrm{p}=0.000)$ respectively. (Fig. 2)

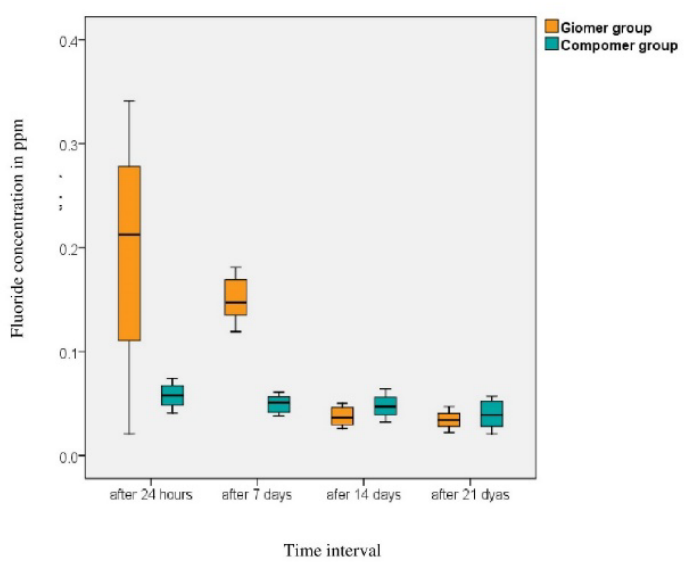

Figure 2: Box and whisker graph of fluoride release (ppm) after different time intervals in the two study groups.

Fluoride concentrations in artificial saliva after topical application of sodium fluoride varnish on teeth restored either with Giomer or Compomer in different time intervals; 24hours, 7days, 14days and 21days were statistically significant different $(\mathrm{p}=0.000)$.

Comparison between the two groups after recharging with topical fluoride in each time interval separately indicated that there was no statistical significant difference in fluoride concentration between the two materials ( $p$ $>0.125$ ). (Fig. 3) 


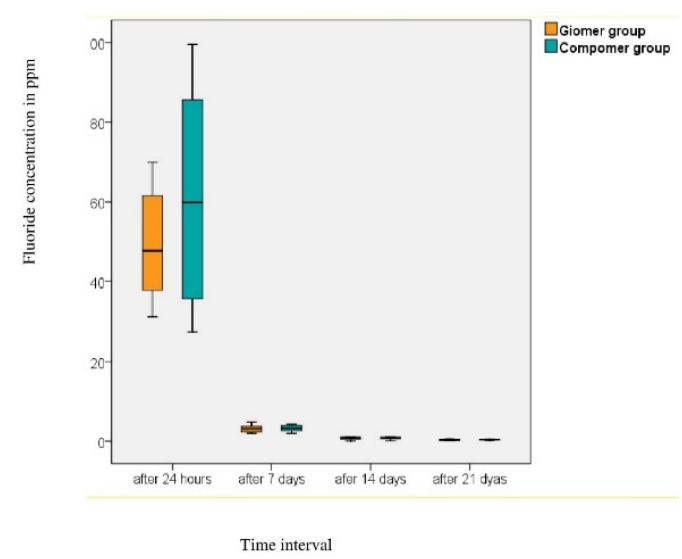

Figure 3: Box and whisker graph of fluoride re-release (ppm) after different time intervals in the two study groups.

Comparison between amount of fluoride released in the artificial saliva of groups I and II after 21 days and its amount 21 days after recharging indicated that there was a statistically significant increase in fluoride amount 21 days after recharge than before for both materials $(p=0.000)$. (Table III).

Table 3: Comparison between concentrations of fluoride released in the artificial saliva of groups I and II after 21days and its concentration 21 days after recharging.

\begin{tabular}{|c|c|c|}
\hline & Giomer & Compomer \\
\hline $\begin{array}{c}\text { After 21 days } \\
\text { from the } \\
\text { beginning of the } \\
\text { study (just before } \\
\text { recharge) }\end{array}$ & Min-Max 0.022-.047 & Min-Max 0.021-0.057 \\
\hline Median 0.034 & IQR 0.028-.041 & Median 0.039 \\
On the day 21 & IQR 0.028-0.052 \\
after recharge & Min-Max 0.097-0.551 & \\
& Median 0.346 & Min-Max 0.214-0.525 \\
& IQR $0.199-0.445$ & Median 0.405 \\
& & IQR 0.311-0.487 \\
\hline Test of & & \\
significance & & \\
P value & & \\
\hline
\end{tabular}

(1)

*: statistically significant difference $(\mathrm{p}<0.0125)$

\section{DISCUSSION}

Compomer (polyacid modified composite) is a hybrid aesthetic restorative material that contains the glass ionomer fillers within the composite resin matrix. It became clinically favorable due to their improved physical and mechanical properties compared to glass ionomer restorative material (11) in addition to their ability to act as a reservoir for direct fluoride release to susceptible tooth surfaces in high caries risk children (21).

Giomer (Beautifil II) is a new category of hybrid aesthetic restorative material. It contains surface prereacted glass (S-PRG) ionomer filler particles which provide the properties of fluoride release of glass ionomer in addition to the superior physical properties of resin composite as claimed by manufacturer (15). The current study found an interest to compare the fluoride release of prereacted glass ionomer composite-Giomer (Beautifil II) to polyacid modified composite-Compomer (Dyract XP) restorations in primary teeth and to evaluate their recharging ability with topical fluoride application.

Many methods have been employed to estimate the amount of fluoride release such as spectrophotometry, ion chromatography, fluoride ion-specific electrodes and capillary electrophoresis. Ion specific electrode with an ion analyzer was used in this study because it is simple, inexpensive and does not require the use of complex laboratory equipment. Moreover, it gives an accurate and direct estimate of the free fluoride present in solution (22).

The current study showed that Giomer released the highest amount of fluoride after 24 hours which remains relatively constant for one week. In the second week, Steady decline of release was significant. However, fluoride release became relatively constant again till the $21^{\text {st }}$ day. This finding was in agreement with Choudhary et al in 2015 (23). They evaluated the release of fluoride ions from GIC, Giomer and Compomer before and after recharge with topical fluoride gel. Giomer showed a steady decrease in release of fluoride with time rather than sharp decline like GIC. Result of study conducted by Yap et al in 2002 (22), who studied the short term fluoride release of GIC, Giomer and Compomer was also in agreement to the results of the present study. They found that fluoride release of Giomer at day seven was significantly greater than at day 21. In contrary, Harhash et al in 2017 (24), measured the amount of fluoride released from Giomer and two types of composite after 1day, 7 days and 4 weeks and found a significant decrease of fluoride release from day 1 to day 7 in Giomer.

Statistical analysis of the present study indicated that Compomer showed a different pattern of fluoride release in comparison to Giomer. This difference is mainly related to their setting reaction (22). Greatest fluoride release in Compomer was after 24 hours. However it decreased significantly through the first week and after that it became relatively constant till the $21^{\text {st }}$ day. This result was in agreement with Cildir et al (2005) (25) and Ahn et al (2011) (26). This is in contrary to Neelakantan et al, their study in 2011 (27) showed that Compomer had no initial fluoride "burst" effect but lower constant level of fluoride release than conventional GIC from the first day throughout the 28 days study period.

Results of the current study showed that Giomer released significantly higher amount of fluoride than Compomer during the first week. This is in accordance with Bansal and Bansal (2017) (28). On contrary, study of Mousavinasab et al in 2009 (29) showed that Giomer released significantly less fluoride than Compomer on the $1^{\text {st }}$ week. However, in the present study, Compomer released significantly higher amount of fluoride than Giomer on the $14^{\text {th }}$ till the $21^{\text {st }}$ day. This finding was in agreement with Yap et al in 2002 (22). In contrast, Al-Naimi et al showed in 2008 (30) that Fluoride release of Giomer and Compomer in natural saliva with normal $\mathrm{pH}$ was comparable and no statistically significant difference existed between them. Moreover, Gui et al in 2015 (17) proved in their study that Giomer and Compomer released comparable amount of fluoride.

The observed fluoride releasing pattern demonstrated by Giomer and Compomer can be attributed to the dissolution 
of their fluoride containing glass filler particles into their resin matrix and surrounding media.

Compomer contains a mixture of monomers and reactive glass fillers. Initial setting is occurring through photopolymerization, followed by acid base reaction that arises from water sorption. Therefore after curing of Compomer and before contact with water the fluoride is not free, but bound in the filler particles which are enclosed in the polymerized matrix. Hence, in the first phase of setting, Compomer completely behave like composite (29).

Giomer contains surface pre-reacted glass ionomer filler which initially reacts with poly acrylic acid in an acid base reaction prior to incorporation into the resin matrix. Giomer has additional sources of fluoride for release; the fluoride complexes within their glass ionomer hydrogel of prereacted glass particles which might explain its significant continuous high level of fluoride release in the first week. In addition to that, acidified water within the hydrogel layer facilitates more fluoride release through additional dissolution of the fluoride containing unreacted glass core (31, 32).

Professionally applied fluoride varnish is a common caries preventive measure used in pediatric dentistry (33). It was used to recharge the tested restorative materials in a way similar to the clinical situation. The ability of the topical fluoride agent for recharging a material is dependent on the dose, frequency, concentration and duration of application of this agent (28). The fluoride re-release that occurs immediately after recharge is induced by superficial effect of the topical fluoride while during the subsequent days release is attributed to its ability to diffuse through the materials' pores and stored to be re-released (34).

The results obtained in the present study showed that both Giomer and Compomer were able to be recharged with topical fluoride with no statistically significant difference between them. These findings were on agreement with the studies performed by Rohani et al in 2009 (35) and Dhull et al in 2011 (16). The latter found that increasing fluoride exposure significantly increased fluoride release from Giomer and Compomer. However, in contrary to the results of this study, they found that Giomer showed comparatively greater fluoride recharge than Compomer with statistically significant difference. In contrast with the results of our study, Gururaj et al in 2013 (36) compared in vitro the fluoride re-release from 5 different aesthetic restorative materials including Giomer and Compomer. They concluded that recharging capability of Compomer was higher than Giomer.

Results obtained from the current study indicated the importance of topical fluoride application. Giomer and Compomer could be recharged with fluoride. Concentrations of fluoride in the artificial saliva after 21 days of recharge are significantly greater than its concentrations just before application of the topical fluoride varnish. Contradictory results to the present study have been reported in 2012 by Abdul Quader et al (37) and Choudhary et al in 2015 (23), Their results indicated that fluoride release increased substantially 1 day after recharge but declined rapidly to near the base line level after 2 to 3 days. This contradiction may be due to the use of different methodology.

In 2014, according to Jingarwar (38), the ability of a material to exhibit fluoride recharge depends on its ability to retain fluoride. The glass ionomer phase incorporated within both tested materials is responsible for their fluoride recharge. The relatively hydrophobic nature of the resin matrices of the two materials implicates the glass ionomer as the key reason for the additional recharge. The hydrogel of PRG particles in Giomer exhibits a high permeability and porosity which consequently provides Giomer with areas within its structure capable of greater fluoride uptake (39).

Giomer showed an initial significant fluoride release for 7 days which may have clinical implications as it will reduce the viability of bacteria that may have been left in the inner carious dentine and induce enamel, dentin remineralization. Moreover the amount of fluoride ion released for both material decreased overtime. Thus the recharging ability of both materials is of great importance. It helps to maintain an increased level of fluoride ion around restoration which is essential for children with high caries risk (5).

A possible limitation of the present study is that the dynamic nature of conditions found actually in the oral cavity such as salivary flow characteristics, presence of plaque, oral hygiene and dietary habits utilized by the patient which can lead to results that may be different from what have been proven in the current study. Thus further studies employing clinical trials are important.

Within the limitations of the study and based on the previous data, the first tested null hypothesis can be rejected as there was statistical significant difference in fluoride release between Giomer and Compomer. The second tested null hypothesis can be accepted.

\section{CONCLUSION}

Fluoride release (ppm) was found to be higher in Giomer (Beautifil II) when compared with Compomer (Dyract XP) in the first week after restoration. Fluorides re-release from Giomer (Beautifil II) and Compomer (Dyract XP) was high when recharging with professionally applied fluoride varnish. Giomer (Beautifil II) showed comparable fluoride recharge with Compomer (Dyract XP). Giomer restorative material could be considered a suitable restoration of primary teeth in high caries risk children.

\section{CONFLICT OF INTEREST}

The authors declare that they have no conflicts of interest.

\section{ACKNOWLEDGEMENT}

My thanks and gratitude to all the faculty members of Pediatric Dentistry department, Dental Biomaterials department and the Institute of Graduate studies and Research for their help during conduction of this study.

\section{REFERENCES}

1. Yengopal V, Harnekar SY, Patel N, Siegfried N. Dental fillings for the treatment of caries in the primary dentition. Cochrane Database SystRev 2009; 15:120-46.

2. Garcez RM, Buzalaf MA, De Araújo PA. Fluoride release of six restorative materials in water and ph-cycling solutions. J Appl Oral Sci 2007; 15:406-11.

3. Murray JJ, Rugg-Gunn AJ, Jenkins GN. Fluorides in caries prevention. J Dent 1993; 21:259-320.

4. John WN. Fluoride-Releasing Dental Restorative Materials: An Update. Balk J Dent Med 2014; 18:60-9.

5. American Academy of Pediatric Dentistry. Guideline on fluoride therapy. Pediatr Dent 2014; 35:165-8. 
6. Hicks J, Garcia-Godoy F, Donly K, Flaitz K, Calif J. Fluoride-releasing restorative materials and secondary caries. J Calif Dent Assoc 2003; 31: 229-45.

7. Berg JH, Croll TP. Glass ionomer restorative cement systems: an update. Pediatr Dent. 2015; 37:116-24.

8. Tiwari S, Nandlal B. Effect of nano-filled surface coating agent on fluoride release from conventional glass ionomer cement: an in vitro trial. J Indian Soc Pedod Prev Dent 2013; 31; 91-5.

9. Mungara J, Philip J, Joseph E, Rajendran S, Elangovan A, Selvaraju G. Comparative evaluation of fluoride release and recharge of pre-reacted glass ionomer composite and nanoionomeric glass ionomer with daily fluoride exposure: An in vitro study. J Indian Soc Pedod Prev Dent 2013; 31:2349.

10. Keles S, Yilmaz Y, Sezen O. Microtensile Bond Strength of Polyacid-modified Composite Resin to Irradiated Primary Molars. JCDP 2018; 19:189-95.

11. Hugar SM et al. Comparative Assessment of Conventional Composites and Coloured Compomers in Permanent Molars of Children with Mixed Dentition: A Pilot Study. JCDR 2017;11:69-72

12. Burke FM, Ray N J, McConnell R J. Fluoride-containing restorative materials .Int Dent J 2006; 56:33-43.

13. Arora V, Bogra P. Giomer - A new hybrid aesthetic restorative material. J Conserv Dent 2002; 5:149-55.

14. Ikemura K, Tay FR, Kouro Y, Endo T, Yoshiyama M, Miyai K, Pashley DH. Optimizing filler content in an adhesive system containing pre-reacted glass-ionomer fillers. Dent Mater 2003; 19:137-46.

15. Walia R, Jasuja P, Verma KG, Juneja S, Mathur A, Ahuja L. A comparative evaluation of microleakage and compressive strength of Ketac Molar, Giomer, Zirconomer, and Ceram-x: An in vitro study. J Indian Soc Pedod Prev Dent 2016; 34:280-4.

16. Dhull KS, Nandlal B. Effect of low-concentration daily topical fluoride application on fluoride release of giomer and compomer: an in vitro study. J Indian Soc Pedod Prev Dent 2011; 29:39-45.

17. Gui Y, Zhao X, Li S, Tang L, Gong X. Fluoride release and recharge properties of six restorative materials. Zhonghua Kou Qiang Yi Xue Za Zhi 2015; 50:28-32.

18. Daniel W, Cross C. Multiple Regression and Correlation. In: Biostatistics. A foundation for analysis in the health science. 10th edition, NewYork: John Wiley and sons, Inc; 2013.pp 480-530.

19. Waggoner WF. Restorative Dentistry for the Primary Dentition. In: Casamassimo PS, Mctigue DJ, Fields HW, Nowak AJ. Pediatric Dentistry; Infancy Through Adolescence .5th edition ,St .Louis;EL Sevier Saunders;2013.pp 324-6.

20. Miller D. Ion-Selective Electrode Determination of Fluoride Ion. Chem Lab Manual .California State University 2011; 321:23-7.

21. Guedes AP et al. Effect of Fluoride-Releasing Adhesive Systems on the Mechanical Properties of Eroded Dentin. Braz Dent J 2016; 27:153-9.

22. Yap AU, Tham SY, Zhu LY \& Lee HK Short-term fluoride release from various aesthetic restorative materials. Oper Dent 2002; 27: 259-65.

23. Choudhary HV, Tandon S, Rathore M, Gopal K, Tiwari N. Fluoride release and uptake by glass ionomer cements, Polyacid modified composite resin and giomer- an in vitro Assessment. IJOCR 2015; 3:68-74.
24. Harhash AY, ElSayad II, Zaghloul AGS. A comparative in vitro study on fluoride release and water sorption of different flowable esthetic restorative materials. Eur J Dent 2017; 11:174-9.

25. Cildir SK, Sandalli N. Fluoride release/uptake of glassionomer cements and polyacid-modified composite resins. Dent Mater J 2005; 24:92-7.

26. Ahn SJ, Lee SJ, Lee DY, Lim BS. Effects of different fluoride recharging protocols on fluoride ion release from various orthodontic adhesives. J Dent 2011; 39:196-201.

27. Neelakantan P, John S, Anand S, Sureshbabu N, Subbarao C. Fluoride release from new glass-ionomer cement. Oper Dent 2011; 36:80-5.

28. Bansal R, Bansal T. A Comparative Evaluation of the Amount of Fluoride Release and Re-Release after Recharging from Aesthetic Restorative Materials: An in vitro Study. JCDR. 2017; 11:11-4.

29. Mousavinasab SM, Meyers I. Fluoride release by glass ionomer cements, compomer and giomer. Dent Res J (Isfahan) 2009; 6:75-81.

30. Al-Naimi OT1, Itota T, Hobson RS, McCabe JF. Fluoride release for restorative materials and its effect on biofilm formation in natural saliva. J Mater Sci Mater Med 2008; 19:1243-8.

31. Attar N and Turgut MD. Fluoride release and uptake capacities of fluoride-releasing restorative materials. Oper Dent 2003; 28: 395-402.

32. Naoum S, Ellakwa A, Martin F, Swain M. Fluoride release, recharge and mechanical property stability of various fluoride-containing resin composites. Oper Dent 2011; 36:422-32.

33. Weyant RJ et al. Topical fluoride for caries prevention: Executive summary of the updated clinical recommendations and supporting systematic review. JADA 2013; 144:1279-91.

34. Jingarwar MM, Pathak A, Bajwa NK, Sidhu HS. Quantitative Assessment of Fluoride Release and Recharge Ability of Different Restorative Materials in Different Media: An in Vitro Study. J Clin Diagn Res 2014; 8:31-4.

35. Rohani R, Nicholson JW. The interaction of Polyacidmodified composite resins (“compomers”) with aqueous fluoride solutions. J Appl Oral Sci 2009; 17:216-19.

36. Gururaj M, Shetty R, Nayak M, Shetty S, Kumar CV. Fluoride releasing and uptake capacities of esthetic restorations. J Contemp Dent Pract 2013; 14:887-91.

37. Abdul Quader S M, Shamsul Alam M, Bashar A K M, Abdul Gafur, Al-Mansu M A. Compressive Strength, Fluoride Release and Recharge of Giomer. UpDCJ 2012; 2:28-37.

38. Attin T, Buchalla W, Siewert C,Hellwig E. Fluoride release/uptake of polyacid-modified resin composites (compomers) in neutral and acidic buffer solutions J Oral Rehabil1999; 26:388-93.

39. Preston AJ, Agalamanyi EA, Higham SM, Mair LH. The recharge of esthetic dental restorative materials with fluoride in vitro-two years results. Dent Mater2003; 19; 327. 